\title{
The efficiency of Trifolium pratense seed extract against the deleterious effects caused by nicotine in the liver of rat
}

\author{
Hasan EL Sayyad ${ }^{1}$; Amna M. Mostafa ${ }^{2}$ and Zainab Kamal ${ }^{2}$ \\ 1- Department of Zoology, Faculty of Science, EL Mansora University \\ 2- Department of Zoology, Faculty of Science, South Valley University
}

\begin{abstract}
The aim of this study was to determine the adverse effects of maternal nicotine treatment during pregnancy and on rat liver of newborns. Also to determine a protective effect of Trifolium Pratense seed extract against the deleterious effects of nicotine. All groups were treated from the $6^{\text {th }}$ day of gestation till parturition. The dams and their newborns were sacrificed and piece of liver from each subjected to the histological studies. The morphological findings of the newborns revealed marked signs of retardation in growth with malformations including superficial haematoma, shortened neck region, kinky tail, uni-and bilateral deformity of both fore and hind limbs and abnormal skin. The histopathological studies on the liver of the maternal nicotine treated and their newborns revealed disruption of hepatic cords arrangement. Blood sinusoids were dilated. The hepatic tissue exhibited inflammatory cells with signs of early cellular granulomatous lesions. Some hepatocytes obliterated and others with karyolysis nuclei. Kupffer cells become hypertrophied and dispersed throughout the hepatic cords. Fatty vacuoles and infiltrations were detected in many of the hepatocytes.

The magnitude of these changes was dose dependent. TPSE supplementation was found to ameliorate an prevent the adverse effects of maternal nicotine treatment morphologically and histologically.
\end{abstract}

Key Words: Trifolium Pratense seed (TPSE) - Liver - Mother Rat - Nicotine - Newborns. 